\title{
Are animals a source of Stenotrophomonas maltophilia in human infections? Contributions of a nationwide molecular study
}

\author{
Aurélie Jayol ${ }^{1,2,3} \cdot$ Camille Corlouer $^{1} \cdot$ Marisa Haenni $^{4} \cdot$ Mélanie Darty $^{5} \cdot$ Karine Maillard $^{6} \cdot$ Marine Desroches $^{1}$. \\ Brigitte Lamy $^{7,8} \cdot$ Estelle Jumas-Bilak ${ }^{9,10}$ • Jean-Yves Madec ${ }^{4}$. Jean-Winoc Decousser ${ }^{1,11}$
}

\begin{abstract}
Stenotrophomonas maltophilia $(\mathrm{Sm})$ is an archetypal environmental opportunistic bacterium responsible for health careassociated infections. The role of animals in human $S m$ infections is unknown. This study aims to reveal the genetic and phylogenetic relationships between pathogenic strains of $S m$, both animal and human, and identify a putative role for animals as a reservoir in human infection. We phenotypically and genotypically characterized $61 \mathrm{Sm}$ strains responsible for animal infections (mainly respiratory tract infections in horses) from a French nationwide veterinary laboratory network. We tested antimicrobial susceptibility and performed MLST and genogrouping using the concatenation of the seven housekeeping genes from the original MLST scheme. Excluding the eight untypeable strains owing to the lack of gene amplification, only 10 out of the 53 strains yielded a known ST (ST5, ST39, ST162, ST8, ST27, ST126, ST131). The genogroup distribution highlighted not only genogroups (genogroups 5 and 9) comprised exclusively of animal strains but also genogroups shared by human and animal strains. Interestingly, these shared genogroups were primarily groups 2 and 6, which have previously been identified as the two most frequent genogroups among human-pathogenic Sm strains, especially among respiratory pathogens. The antimicrobial susceptibility testing underlined the presence of acquired resistance: 18.8 and $7.5 \%$ of the tested isolates were resistant to the sulfonamide-trimethoprim combination and ciprofloxacin, respectively. Animal strains of $S m$ shared phylogenetic traits with some of the most successful human strains. The exact relationships between the human and animal strains, and the genetic support of these common traits, need to be determined.
\end{abstract}

This work was presented in part at the 25th European Congress of Clinical Microbiology and Infectious Diseases (ECCMID), poster P0137, Copenhagen, Denmark, 25-28 April 2015.

Jean-Winoc Decousser

jean-winoc.decousser@hmn.aphp.fr

Laboratory of Bacteriology and Infection Control, Department of Microbiology, Assistance Publique-Hôpitaux de Paris, University Hospital Henri Mondor, 94000 Créteil, France

2 Emerging Antibiotic Resistance Unit, Medical and Molecular Microbiology, Department of Medicine, University of Fribourg, Fribourg, Switzerland

3 INSERM European Unit (LEA-IAME Paris, France), University of Fribourg, Fribourg, Switzerland

4 Unité Antibiorésistance et Virulence Bactériennes, ANSES, Lyon, France

5 Next Generation Sequencing Platform, University Hospital Henri Mondor, Assistance Publique-Hôpitaux de Paris, Créteil, France

6 Laboratoire Labeo Frank Duncombe, Caen, France
7 Department of Bacteriology, Archet 2 Hospital, Nice Academic Hospital, Nice, France

8 INSERM U1065, C3M, Team 6, Nice, France

Infection Control Department, Montpellier University Hospital, Montpellier, France

10 UMR5569 HydroSciences Montpellier, Equipe «Pathogènes Hydriques Santé Environnements», Faculté de Pharmacie, 15 Avenue Charles Flahault BP 1449134093 Montpellier UMR 5119 ECOSYM, Equipe Pathogènes et Environnements, U.F.R. des Sciences Pharmaceutiques et Biologiques, Université Montpellier 1, Montpellier, France

11 EA 7380 Dynamyc Université Paris-Est Créteil (UPEC), Ecole nationale vétérinaire d'Alfort (EnvA), Faculté de Médecine de Créteil, 8 rue du Général Sarrail, 94010 Créteil, France 


\section{Introduction}

Stenotrophomonas maltophilia $(\mathrm{Sm})$ is an archetypical opportunistic bacterium, infecting immunocompromised patients [1]. The natural reservoir of $S m$ is the environment, particularly the rhizosphere and freshwater. The genetic diversity of $\mathrm{Sm}$ strains has been well studied [1,2]. The hypothesis of the co-existence of different phylogenetic groups, among which some are more virulent than others, arose from the first studies using molecular methods [3-5]. Understanding the mechanism of selection of human pathogenic strains from this diversity requires sound molecular approaches and large and diverse samples of strains [6]. The genogroup organization is robust enough to be confirmed by different molecular methods, such as amplified fragment polymorphism (AFLP), gyrB restriction fragment length polymorphism (RFLP) analysis, and multilocus sequence typing (MLST) [3, 4, 7]. We recently applied this last method to a large sample of human strains responsible for health care-associated infections, and we underlined the high prevalence of genogroup 6 and, more surprisingly, genogroup 2 [8]. Unfortunately, the various previously published studies did not include any strains originating from animals, even though $\mathrm{Sm}$ is responsible for colonization and infection in animals, particularly in horses [1, 4, 5, $7,9,10]$. A study of the genetic background of infectious strains from animals can help investigate their putative role as a reservoir for human infections. Additionally, as previously reported, this approach can contribute to the identification of highly virulent genogroups and/or genogroups that are well adapted to anatomical microenvironments such as the respiratory tract $[11,12]$. In the present work, we performed a phenotypic and genetic characterization of a large sample of animal strains from diverse geographical regions and compared them to their human counterparts.

\section{Material and methods}

\section{Strain collection}

Strains were prospectively collected between January 2013 and May 2014 from the Resapath network (https://www. resapath.anses.fr). This established network, driven by the French agency for food, environmental, and occupational health and safety (ANSES, Agence nationale de sécurité sanitaire de l'alimentation, de l'environnement et du travail; www.anses.fr), collects data and strains for epidemiological purposes from veterinary laboratories throughout French regions $[13,14]$. In the present study, $S m$ strains were collected from infected animals and considered relevant pathogens by each participating local laboratory. The strains were then centralized at the laboratory of bacteriology and infection control, University Hospital Henri Mondor
(Créteil, France). Only one strain per animal was included. The following metadata were collected: animal species, geographical location, site of infection, and specimen type.

\section{Strain characterization}

\section{Phenotypic characterization}

Identification at the species level was performed by matrixassisted laser desorption/ionization time-of-flight mass spectrometry (MALDI-TOF MS) (Andromas ${ }^{\circledR}$, Beckmann Coulter, Villepinte, France). The antimicrobial susceptibility of each strain was tested and interpreted using the diskdiffusion method according to the 2013 guidelines of the Antimicrobial Committee of the French Society of Microbiology (http://www.sfm-microbiologie.org). The following compounds were tested: (1) cotrimoxazole (combination trimethoprim-sulfamethoxazole), (2) ciprofloxacin, (3) ticarcillin-clavulanate, and (4) ceftazidime.

\section{Genotypic characterization}

The MLST profile and phylogenetic tree were obtained as previously described [8]. Briefly, the MLST profile was determined using the Kaiser scheme [7]. For each strain, seven housekeeping genes were amplified and sequenced according to the recommendations of the PubMLST website (http:// pubmlst.org/smaltophilia/): (1) atpD, (2) gapA, (3) guaA, (4) mutM, (5) nuoD, (6) ppsA, and (7) recA. The seven allelic sequences of the seven housekeeping genes were then concatenated for each strain (final length: $3591 \mathrm{nt}$ ), and aligned using ClustalW from the MEGA 7.0 software package (http://www.megasoftware.net/). The phylogenetic trees were reconstructed using the PhyML 3.0 software based on the maximum-likelihood principle (http://www.atgc-montpellier. fr/phyml/) and a 100-repetition bootstrap analysis, and the FigTree v1.4 software; they were rooted on the corresponding S. rhizophila sequences (DSM14405, RefSeq SAMN02727981). Next to the animal strains, the phylogenetic tree included all the 227 MLST types available on the PubMLST website on September 1, 2017, including the 64 $\mathrm{Sm}$ strains from the original work of Kaiser et al. that defined the 14 genogroups [7]. Because we wanted to perform a more specific comparison between human and animal strains coexisting in the same geographic area, we constructed an additional phylogenetic tree including the previously characterized human strains with the current French animal strains [8]. Finally, the clonalities of the animal strains clustering together were tested by comparing the pulsed-field gel electrophoresis profiles after enzymatic $\mathrm{XbaI}$ macrorestriction, as previously described [15]. 


\section{Statistical analysis}

Statistical analysis was carried out using the $\chi^{2}$ test or the Fisher exact test if appropriate, and a $P$ value of $\leq 0.05$ was considered statistically significant.

\section{Results}

\section{Characteristics of the collected strains}

During the period of study, 61 clinically relevant strains of $\mathrm{Sm}$ were collected from respiratory $(59 / 61)$, cutaneous $(1 / 61)$, and urine (1/61) samples from horses (57/61), dogs $(2 / 61)$, cats $(1 / 61)$, and reptiles (1/61). The distribution of geographical locations is shown in Fig. 1. Regarding the antibiotic susceptibility, $83.9 \%$ of $\mathrm{Sm}$ isolated from animals were susceptible to cotrimoxazole, $53.2 \%$ to the ticarcillin-clavulanate combination, $93.5 \%$ to ciprofloxacin, and $40.4 \%$ to ceftazidime.

\section{ST profiles and distribution}

The MLST profiles were obtained for 53 of the 61 strains; their geographical location distribution is shown in Fig. 1. For the remaining eight strains, the MLST profiles were incomplete because one or several of the targeted housekeeping genes could not be amplified.

Among the typeable strains, ten strains exhibited a previously characterized sequence type (ST): ST5 $(n=2)$, ST39 $(n=2), \operatorname{ST} 162(n=2), \mathrm{ST} 8(n=1), \mathrm{ST} 27(n=1), \mathrm{ST} 126$ $(n=1)$, and ST131 $(n=1)$ (Supplementary Table S1). The remaining 43 strains carried alleles of housekeeping genes that varied from 1 to 40 nucleotides (Supplementary Table S2) and were distributed into 33 newly described STs. All new alleles

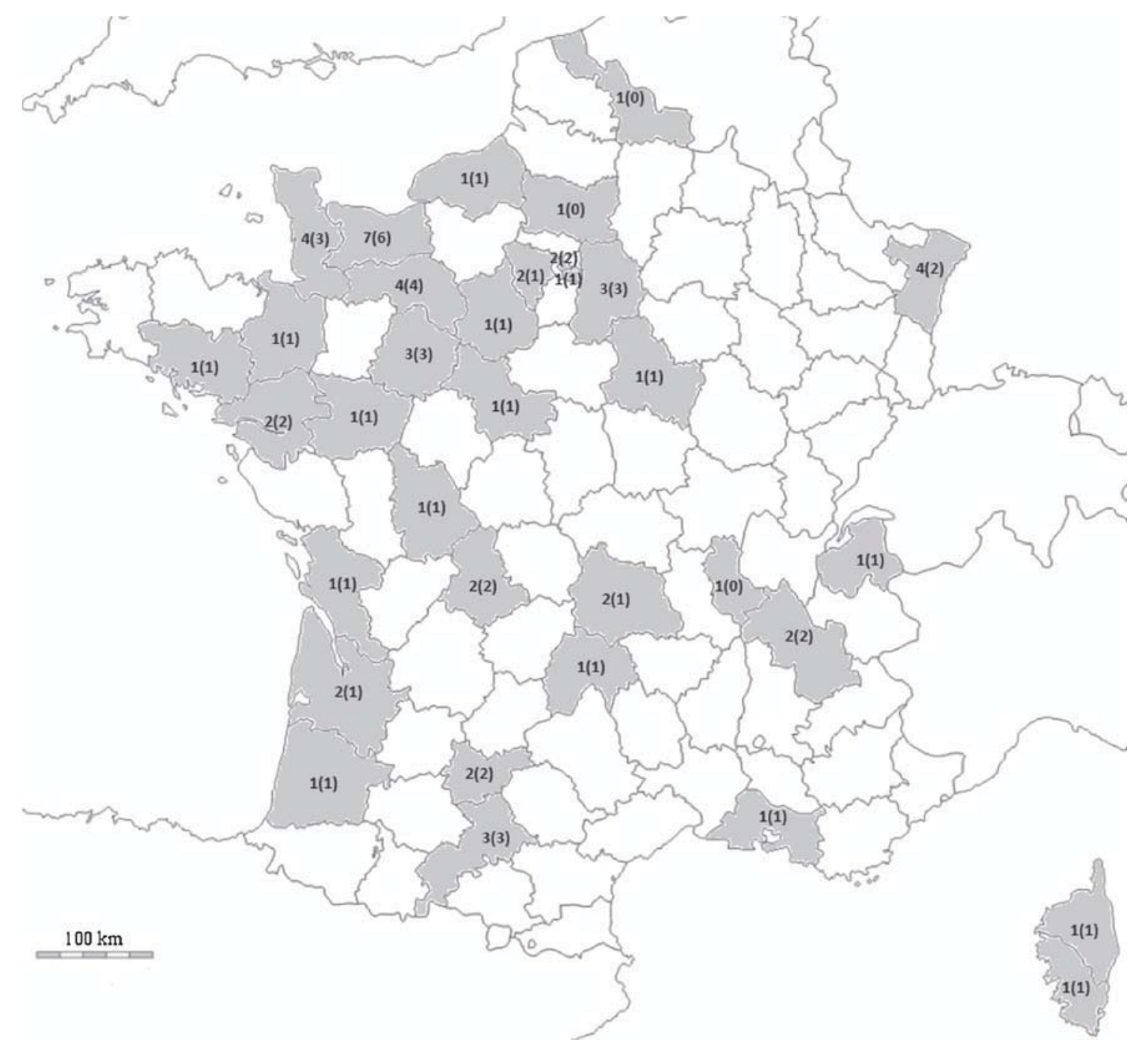

Fig. 1 Location and geographic distribution of the 61 Stenotrophomonas maltophilia animal strains (including the 53 typeable strains). Number in brackets corresponds to the number of typeable strains using the MLST approach 
have been deposed on the Stenotrophomonas maltophilia MLST website (https://pubmlst.org/smaltophilia/).

\section{Genogroup distribution}

The phylogenetic tree reconstructed from the concatenated sequences from the PubMLST database, and the new sequences from the animal strains, is shown in Fig. 2. The affiliated genogroups are also reported in Fig. 2 as well as Supplementary Tables 1 and 2. The phylogenetic tree inferred from the concatenated sequences from the previously published dataset of French human strains, and from the present dataset of animal strains, is shown in Fig. 3 [8]. The PFGE analysis of the animal strains clustering together excluded a common origin of the strains (Supplementary Fig. 1). The genogroup distribution between the human and animal groups of strains is shown in Table 1. The percentage of strains that could not be classified was substantial and non-different in the two groups (20.8 and $26.3 \%$, respectively). Human strains grouped in two main genogroups ( 2 and 6 ), and the remaining genogroups gathered at most $4 \%$ of the strains. Regarding the animal strains, they were distributed among four main genogroups, the two previously reported (2 and 6) and two other genogroups (5 and 9) that did not included human strains. So, animal strains were significantly more frequent than human strains in genogroups 5 and $9(p<0.001)$. In contrast, human strains were more frequent in genogroup 6 $(p=0.001)$.

Fig. 2 Maximum-likelihood tree based on the concatenated sequences of the seven housekeeping gene fragments (3591 nt). The tree shows the structure of the studied Stenotrophomonas maltophilia population that includes (i) the 53 typeable strains of the present study and (ii) all the $227 \mathrm{STs}$ available on the MLST website at the time of analysis, including the 64 S. maltophilia strains from the original work of Kaiser et al. [7]. The horizontal lines represent the genetic distances. The tree was rooted on the corresponding concatenated sequences from Stenotrophomonas rhizophila. The genogroup affiliation according to MLST in the original study is indicated by colored frames [7]

\section{Discussion}

Animals play a significant role in the occurrences of human infectious diseases; in addition to zoonosis, animals can act as reservoirs for human pathogenic bacteria, including multidrug-resistant strains, such as methicillin-resistant Staphylococcus aureus or carbapenem-resistant Enterobacteriaceae $[16,17]$. Moreover, animals can host specific, well-adapted clones that can also become deadly human pathogens, such as S. aureus ST 398 [18]. With regard to opportunistic environmental bacteria, animals can also act as intermediary hosts to allow and favor the selection of subpopulations that exhibit increased human virulence potentials. They may also act as incubators for emerging opportunistic pathogens, through bacterial interactions with shelter organisms that shape bacterial evolution, for example, with invertebrates or rhizosphere microorganisms, as discussed elsewhere [19]. Sm from a wide range of animals, including mammals, fish, and reptiles, has been reported; it has been associated with colonization or with real infections, such as adenitis, abscesses, or respiratory tract infections [1]. Recently, Sm was reported to be responsible for infertility in a dog [20]. The putative role of animals as reservoirs for $\mathrm{Sm}$ has been previously reported [21]. Interestingly, $S m$ was responsible for seven cases of lower airway disease in horses, in the context of chronic coughing [10]. We confirmed this association; a large part of our sample of animal strains originated from the horse respiratory tract. Respiratory tract infections are one of the most frequent clinical presentations of Sm in humans; both

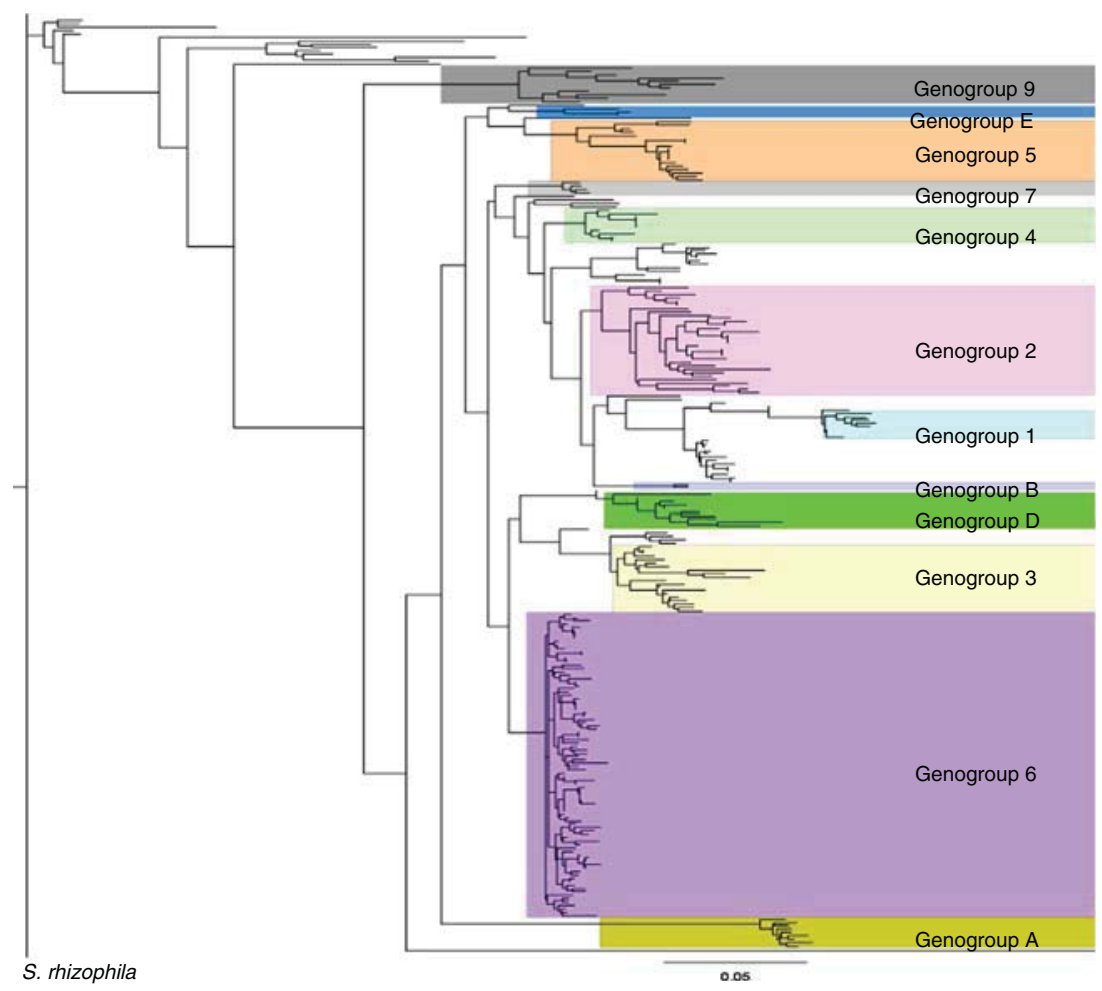




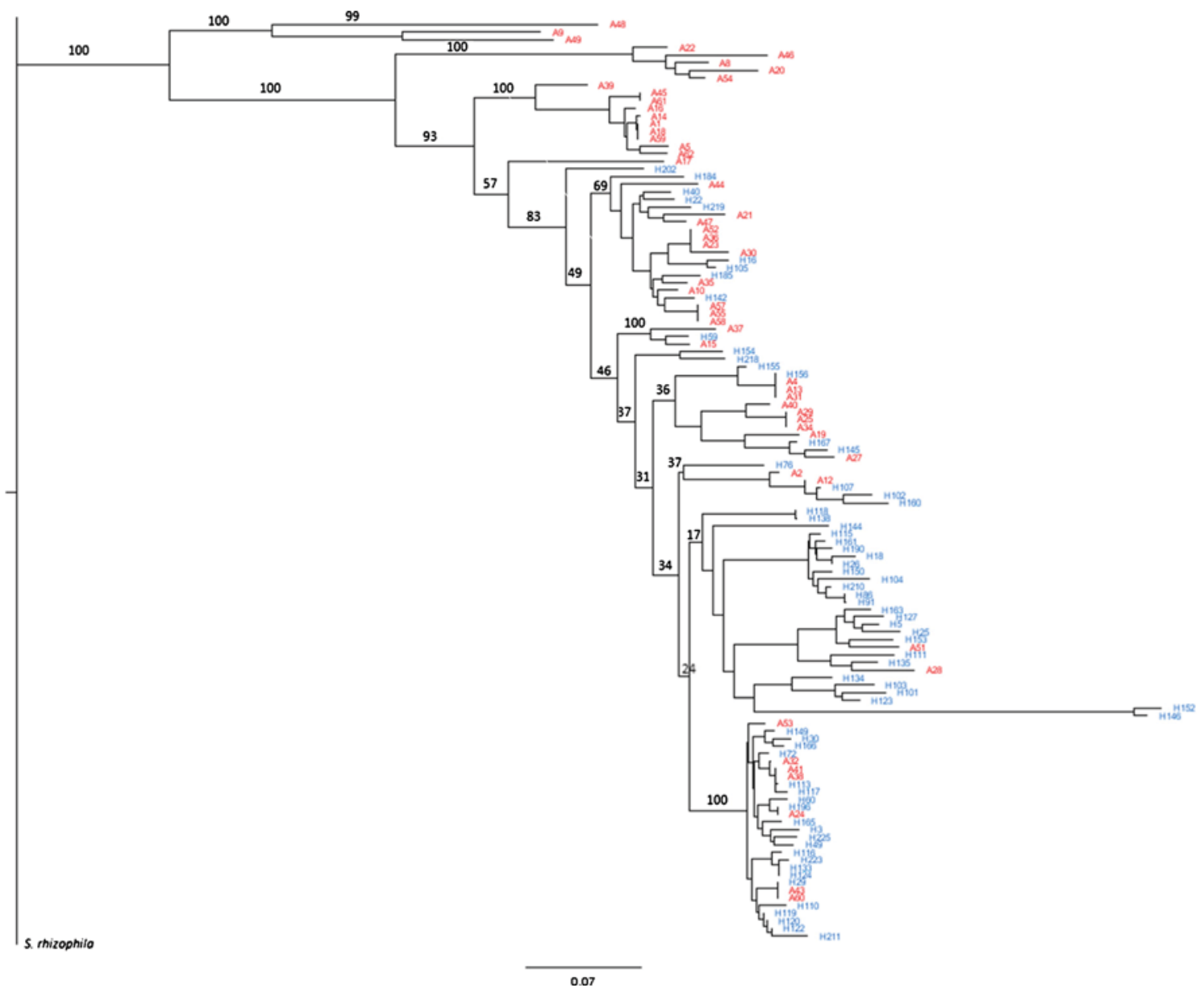

Fig. 3 Maximum-likelihood tree based on the concatenated sequences of the seven housekeeping gene fragments (3591 nt). The tree shows the structure of the studied Stenotrophomonas maltophilia population that includes (i) the 53 typeable animal strains of the present study (colored in red, strain's name beginning with "A") and (ii) the 80 human strains

previously described STs (colored in blue, strain's name beginning with " $\mathrm{H}$ ") [8]. The horizontal lines represent the genetic distance. The tree was rooted on the corresponding concatenated sequences from Stenotrophomonas rhizophila

acute and chronic infections have been reported, in immunocompromised and cystic fibrosis patients, respectively [1]. The study of animal strains could also contribute to the characterization of phenotypic properties or a genetic background dedicated to some types of respiratory tract infections, as previously proposed [4, 22]. In addition to ordinary MLST, which addresses the high genetic diversity in Sm, the description of the genogroups has led to the drawing of a phylogenetic organization that could combine or separate strains from human and environmental origins $[7,23]$. Nevertheless, to the best of our knowledge, this approach has never been applied on strains of animal origin. As reported for human strains, the MLST of animal strains was not very informative: the first 8 out of $61(13.1 \%)$ of the strains were untypeable due to putative mutations on the binding site of the primers essential for gene sequencing [8]. Moreover, only 10/53 (18.9\%) of the remaining strains yielded a known ST. This is further evidence of the high genetic diversity of Sm [2]. The ST5 represented 2 out of 10 strains; this ST has been previously identified in

human strains from different countries (France, Germany, Austria, Korea) $[7,8,24]$. The other STs were not as frequently reported as ST5. The genogroup determination that is based on the concatenation and alignment of the sequences corresponding to the seven housekeeping genes is an elegant and efficient approach to overcome the genetic diversity of $\mathrm{Sm}$ and the relative poverty of the corresponding MLST database [7, 23]. The genogroup distribution among animal strains clearly highlights the predominance of genogroup 6; this genogroup included 6 out of the 10 strains that harbored a previously described ST. In addition to the ST identification, the genogroups 2 and 6 , the two most frequently encountered genogroups responsible for infections in humans, were comprised 26.4 and $13.2 \%$ of animal strains [8]. The presence of animal and human strains in the same genogroups suggest the possibility of (i) strain exchange between humans and animals or (ii) gene exchange through horizontal gene transfer between strains from the same genogroup, as reported by Cerezer et al. for human and environmental strains [9]. 
Table 1 Genogroup distribution among animal and human strains

\begin{tabular}{lcc}
\hline \multirow{2}{*}{ Genogroup } & \multicolumn{2}{l}{ Genogroup distribution $\%(n / N)$} \\
\cline { 2 - 3 } & \multicolumn{1}{c}{ Animal strains $(N=53)$} & Human strains $(N=80)$ \\
\hline 1 & $0.0 \%(0 / 53)$ & $3.8 \%(3 / 80)$ \\
2 & $26.4 \%(14 / 53)$ & $16.3 \%(13 / 80)$ \\
3 & $3.8 \%(2 / 53)$ & $1.3 \%(1 / 80)$ \\
4 & $5.7 \%(3 / 53)$ & $2.5 \%(2 / 80)$ \\
5 & $18.9 \%(10 / 53)$ & $0.0 \%(0 / 80) *$ \\
6 & $13.2 \%(7 / 53)$ & $41.3 \%(33 / 80) *$ \\
7 & $0.0 \%(0 / 53)$ & $2.5 \%(2 / 80)$ \\
9 & $9.4 \%(5 / 53)$ & $0.0 \%(0 / 80) *$ \\
$\mathrm{~A}$ & $0.0 \%(0 / 53)$ & $2.5 \%(2 / 80)$ \\
$\mathrm{D}$ & $1.9 \%(1 / 53)$ & $3.8 \%(3 / 80)$ \\
E & $0.0 \%(0 / 53)$ & $0.0 \%(0 / 80)$ \\
Unclassified & $20.8 \%(11 / 53)$ & $26.3 \%(21 / 80)$ \\
\hline
\end{tabular}

$n$ number of strains belonging to the genogroup, $N$ total number of animal or human strains

*Statistically significant difference between human and animal distribution $(p<0.05)$

Moreover, these genogroups included a large number of the strains responsible for respiratory tract infections in both human and animals [8]. Therefore, our study supports the putative role of animals as reservoirs for human infections, or similar mechanisms of infection of the respiratory tract from a common environmental source. As discussed later, we could not rule out the possibility of the colonization of domestic animals through close contact with their owners. In contrast, two genogroups were characterized as being composed exclusively of animal strains: genogroups 5 and 9. Kaiser et al. reported similar results with three genogroups specific to environmental strains: genogroups 5, 8, and 9 [7]. In our previously published work, we failed to connect human strains to these genogroups. Interestingly, we noticed that animal strains could be genetically connected to these genogroups.

In addition to the genetic considerations about the dynamics of $S m$ clones between the environment, animals, and humans, our findings contribute to the debate about the putative origins of $S m$ strains infecting patients. Indeed, it has been established that $\mathrm{Sm}$ colonization can occur outside the clinical setting, preceding endogenous infection [1]. In addition to environmental sources such as freshwater, we suggest that patients can be colonized through contact with animals. This could be of additional interest during the investigation of the few cases of community-acquired $\mathrm{Sm}$ infections [25].

The antimicrobial susceptibilities of the animal strains underline the presence of acquired resistance to the first- and second-line agents, in proportions similar to or higher than those of their human counterparts. For example, the cotrimoxazole resistance rate was $18.8 \%$ for animal strains and $5.0 \%$ for human strains in our previously published work. Owing to the low number of strains, these results should be cautiously interpreted. Ciprofloxacin- and trimethoprimsulfamethoxazole-resistant strains of $\mathrm{Sm}$ from animal infections have been previously reported [26]. In France, the nature and volume of antibiotics consumption in animals, including trimethoprim, sulfonamides, and fluoroquinolones, could promote the selection of resistant strains [27]. Our study of samples originating mainly from horses suffering from airway infections cannot exclude the possibility that the animals were exposed to antibiotics before specimen collection, as reported in the original study of Winther et al. [10].

Our work has several limitations. First, the strains originating from horses dominate the sample of animal strains. Our findings must be compared to a larger and more diverse sample of animal strains, including wildlife, livestock and companion animals, and mammalian and non-mammalian animals. Nevertheless, the four strains that originated from animals other than horses, grouped in two well-established genogroups that we found to be as preponderant in humans, i.e., genogroup 2 (one strain from cats) and genogroup 6 (two strains from dogs and one strain from reptiles). Moreover, the geographic distribution was large, including a significant part of the French countryside. Second, the Sm strains were detected from animals that have contact with humans; this could corrupt the phylogenetic analysis by including strains of human origin that colonized animals. Although the sample of strains must include more animal species that do not have contact with humans, our results show that we characterized two genogroups exclusively composed of strains of animal origin (genogroups 5 and 9). Our study would benefit from the inclusion of a large panel of environmental strains of various origins to evaluate the relationships between animal and environmental strains. Finally, the number of strains was quite low; the hypothesis of the putative role of animal in human infections due to $\mathrm{Sm}$ must be confirmed by a larger study. To date, our work could be considered as a proof of concept of the putative contribution of animals in the human epidemiology of Sm infections.

In the near future, the new technical and phylogenetic approaches combining whole-genome sequencing and coregenome MLST will open new opportunities to overcome the technical difficulties of sequencing a limited number of housekeeping genes from a highly genetically diverse bacterial species such as $S m$. In addition to phylogenetic improvements, the availability of whole-genome data should promote the identification of coding regions associated with both human and animal pathogenicity, especially with regard to the capacity to trigger respiratory tract infections.

In conclusion, our work is the first to position animal strains in the genetic phylogeny of Sm. Although some genogroups seem to be exclusively related to animal strains, the sharing of several genetic backgrounds by human and animal strains is of particular interest. The exact role of animals in the epidemiology 
and physiopathology of human $S m$, if it exists, must be determined by large, whole-genome-sequencing-based studies.

Acknowledgments The authors would like to thank the veterinary laboratories participating to the RESAPATH network and Professor HenriJean Boulouis (Ecole nationale vétérinaire d'Alfort, France).

\section{Compliance with ethical standards}

Conflict of interest The authors declare that they have no conflicts of interest.

Ethical approval Not applicable

Informed consent Not applicable

\section{References}

1. Brooke JS (2012) Stenotrophomonas maltophilia: an emerging global opportunistic pathogen. Clin Microbiol Rev 25:2-41

2. Valdezate S, Vindel A, Martín-Dávila P, Del Saz BS, Baquero F, Cantón R (2004) High genetic diversity among Stenotrophomonas maltophilia strains despite their originating at a single hospital. J Clin Microbiol 42:693-699

3. Hauben L, Vauterin L, Moore ER, Hoste B, Swings J (1999) Genomic diversity of the genus Stenotrophomonas. Int J Syst Bacteriol 49:1749-1760

4. Coenye T, Vanlaere E, LiPuma JJ, Vandamme P (2004) Identification of genomic groups in the genus Stenotrophomonas using gyrB RFLP analysis. FEMS Immunol Med Microbiol 40: 181-185

5. Minkwitz A, Berg G (2001) Comparison of antifungal activities and $16 \mathrm{~S}$ ribosomal DNA sequences of clinical and environmental isolates of Stenotrophomonas maltophilia. J Clin Microbiol 39:139-145

6. Adamek M, Overhage J, Bathe S, Winter J, Fischer R, Schwartz T (2011) Genotyping of environmental and clinical Stenotrophomonas maltophilia isolates and their pathogenic potential. PLoS One 6(11): e27615. https://doi.org/10.1371/journal.pone.0027615

7. Kaiser S, Biehler K, Jonas D (2009) A Stenotrophomonas maltophilia multilocus sequence typing scheme for inferring population structure. J Bacteriol 191:2934-2943

8. Corlouer C, Lamy B, Desroches M, Ramos-Vivas J, Mehiri-Zghal E, Lemenand O et al (2017) Stenotrophomonas maltophilia healthcare-associated infections: identification of two main pathogenic genetic backgrounds. J Hosp Infect 96:183-188

9. Cerezer VG, Bando SY, Pasternak J, Franzolin MR, Moreira-Filho CA (2014) Phylogenetic analysis of Stenotrophomonas spp. isolates contributes to the identification of nosocomial and community-acquired infections. Biomed Res Int 2014:151405

10. Winther L, Andersen RM, Baptiste KE, Aalbæk B, Guardabassi L (2010) Association of Stenotrophomonas maltophilia infection with lower airway disease in the horse: a retrospective case series. Vet J 186:358-363

11. Aujoulat F, Jumas-Bilak E, Masnou A, Sallé F, Faure D, Segonds C, Marchandin H, Teyssier C (2011) Multilocus sequence-based analysis delineates a clonal population of Agrobacterium (Rhizobium) radiobacter (Agrobacterium tumefaciens) of human origin. J Bacteriol 193:2608-2618

12. Romano S, Aujoulat F, Jumas-Bilak E, Masnou A, Jeannot JL, Falsen E, Marchandin H, Teyssier C (2009) Multilocus sequence typing supports the hypothesis that Ochrobactrum anthropi displays a human-associated subpopulation. BMC Microbiol 9:267
13. Sorbe A, Chazel M, Gay E, Haenni M, Madec JY, Hendrikx P (2011) A simplified method of performance indicators development for epidemiological surveillance networks - application to the RESAPATH surveillance network. Rev Epidemiol Sante Publique 59:149-158

14. Dahmen S, Haenni M, Châtre P, Madec JY (2013) Characterization of blaCTX-M IncFII plasmids and clones of Escherichia coli from pets in France. J Antimicrob Chemother 68:2797-2801

15. Roscetto E, Rocco F, Carlomagno MS, Casalino M, Colonna B, Zarrilli R et al (2008) PCR-based rapid genotyping of Stenotrophomonas maltophilia isolates. BMC Microbiol 8:202

16. Haenni M, Saras E, Châtre P, Médaille C, Bes M, Madec JY et al (2012) A USA300 variant and other human-related methicillin-resistant Staphylococcus aureus strains infecting cats and dogs in France. J Antimicrob Chemother 67:326-329

17. Stolle I, Prenger-Berninghoff E, Stamm I, Scheufen S, Hassdenteufel E, Guenther S et al (2013) Emergence of OXA-48 carbapenemase-producing Escherichia coli and Klebsiella pneumoniae in dogs. J Antimicrob Chemother 68:2802-2808

18. van Belkum A, Melles DC, Peeters JK, van Leeuwen WB, van Duijkeren E, Huijsdens XW et al (2008) Methicillin-resistant and -susceptible Staphylococcus aureus sequence type 398 in pigs and humans. Emerg Infect Dis 14:479-483

19. Aujoulat F, Roger F, Bourdier A, Lotthé A, Lamy B, Marchandin H, Jumas-Bilak E (2012) From environment to man: genome evolution and adaptation of human opportunistic bacterial pathogens. Genes (Basel) 3:191-232

20. Domosławska A, Zduńczyk S, Jurczak A, Janowski T (2017) Stenotrophomonas maltophilia isolated from prostatic fluid as an infertility factor in a male dog. Andrologia 2017. https://doi.org/10. 1111/and.12769

21. Johnson EH, Al-Busaidy R, Hameed MS (2003) An outbreak of lymphadenitis associated with Stenotrophomonas (Xanthomonas) maltophilia in Omani goats. J Vet Med B Infect Dis Vet Public Health 50(2):102-104

22. Pompilio A, Pomponio S, Crocetta V, Gherardi G, Verginelli F, Fiscarelli E et al (2011) Phenotypic and genotypic characterization of Stenotrophomonas maltophilia isolates from patients with cystic fibrosis: genome diversity, biofilm formation, and virulence. BMC Microbiol 11:159

23. Gherardi G, Creti R, Pompilio A, Di Bonaventura G (2015) An overview of various typing methods for clinical epidemiology of the emerging pathogen Stenotrophomonas maltophilia. Diagn Microbiol Infect Dis 81:219-226

24. Cho HH, Sung JY, Kwon KC, Koo SH (2012) Expression of Sme efflux pumps and multilocus sequence typing in clinical isolates of Stenotrophomonas maltophilia. Ann Lab Med 32:38-43

25. Falagas ME, Kastoris AC, Vouloumanou EK, Dimopoulos G (2009) Community-acquired Stenotrophomonas maltophilia infections: a systematic review. Eur J Clin Microbiol Infect Dis 28:719730

26. Ohnishi M, Sawada T, Marumo K, Harada K, Hirose K, Shimizu A et al (2012) Antimicrobial susceptibility and genetic relatedness of bovine Stenotrophomonas maltophilia isolates from a mastitis outbreak. Lett Appl Microbiol 54:572-576

27. Grave K, Torren-Edo J, Muller A, Greko C, Moulin G, Mackay D et al (2014) Variations in the sales and sales patterns of veterinary antimicrobial agents in 25 European countries. J Antimicrob Chemother 69:2284-2291

DNA Sequences: all new alleles have been deposed on the Stenotrophomonas maltophilia MLST website (https://pubmlst.org/ smaltophilia/) 